\title{
New theoretical coefficient robustness results for FETI-DP
}

\author{
Clemens Pechstein ${ }^{1}$, Marcus Sarkis ${ }^{2}$, and Robert Scheichl ${ }^{3}$
}

\section{Introduction}

In this short note, we present new weighted Poincaré inequalities (WPIs) with weighted averages that allow a robustness analysis of dual-primal finite element tearing and interconnecting (FETI-DP) methods in certain cases where jumps of coefficients are not aligned with the subdomain partition.

Let $\Omega$ be a bounded Lipschitz domain in $\mathbb{R}^{2}$ or $\mathbb{R}^{3}$. We consider the weak form of the scalar elliptic PDE

$$
-\operatorname{div}(\alpha \nabla u)=f \quad \text { in } \Omega,
$$

with a uniformly positive diffusion coefficient $\alpha \in L^{\infty}(\Omega)$ that is piecewise constant with respect to a (possibly rather fine) partitioning of $\Omega$. The discretization by continuous and piecewise linear finite elements (FEs) on a mesh $\mathscr{T}(\Omega)$ leads to the sparse (but in general large) linear system

$$
\mathbf{K u}=\mathbf{f} .
$$

We consider FETI-DP solvers (see Farhat et al. [2001], Mandel and Tezaur [2001], Klawonn et al. [2002]) for the fast (and parallel) solution of this system, and we follow the structure described in [Toselli and Widlund, 2005, Sect 6.4]. To this end, we partition the domain $\Omega$ into non-overlapping subdomains $\Omega_{i}, i=1, \ldots, N$ such that the global mesh $\mathscr{T}(\Omega)$ resolves the interface $\bigcup_{i \neq j} \partial \Omega_{i} \cap \partial \Omega_{j}$. The inter-

Institute of Computational Mathematics, Johannes Kepler University, Altenberger Str. 69, 4040 Linz, Austria, clemens.pechstein@numa.uni-linz.ac.at · Mathematical Sciences Department, Worcester Polytechnic Institute, 100 Institute Road, Worcester, MA 01609-2280, United States, and Instituto de Matemática Pura e Aplicada (IMPA), Brazil, msarkis@wpi . edu - Department of Mathematical Sciences, University of Bath, Bath BA2 7AY, United Kingdom, r.scheichl@maths.bath.ac.uk 
(a)

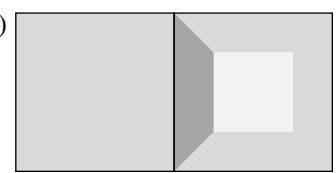

(b)

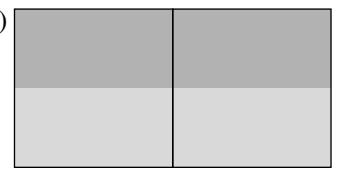

(c)

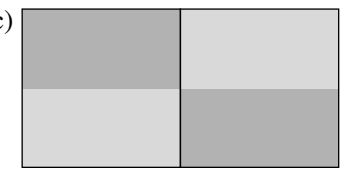

Fig. 1 Different types of coefficient jumps along an edge between two subdomains: (a) across (b) along (c) both across and along.

face itself can be divided into subdomain vertices, edges, and faces (for $d=3$ ), cf. [Toselli and Widlund, 2005, Sect. 4.2].

Without loss of generality, we assume that $\alpha$ is constant on each element of $\mathscr{T}(\Omega)$. Crucially, we do not assume that $\alpha$ is constant on each subdomain. However, we need assumptions on the kind of jumps. Let $\alpha_{i}$ denote the restriction of $\alpha$ to $\Omega_{i}$ and note that it has a well-defined trace in $L^{2}\left(\partial \Omega_{i}\right)$. For each subdomain edge (face) $\mathscr{E}$ on $\Omega_{i}$, we define the weighted average

$$
\bar{v}^{\mathscr{E}, \alpha_{i}}:=\frac{\int_{\mathscr{E}} \alpha_{i} v}{\int_{\mathscr{E}} \alpha_{i}} .
$$

Assumption A1. Whenever two $\Omega_{i}$ and $\Omega_{j}$ share an edge (face) $\mathscr{E}$, the weighted averages of any function $v \in V^{h}(\mathscr{E})$ coincide: $\bar{v}^{\mathscr{E}}, \alpha_{i}=\bar{v}^{\mathscr{E}, \alpha_{j}}$.

A sufficient condition for Assumption A1 is that the coefficient jumps either across or along, but not both at the same time. For an illustration see Figure 1. Our assumptions rules out situations of type (c).

Following [Toselli and Widlund, 2005, Algorithm B], we define the primal space $\widehat{W}_{\Pi}$ spanned by the vertex nodal basis functions at subdomain vertices, the subdomain edge cut-off functions and subdomain face cut-off functions (all of them extended discrete $\alpha$-harmonically from the interface to the subdomain interiors). The dual space $W_{\Delta}$ contains FE functions that are discontinuous across the subdomain interfaces with vanishing $\alpha$-weighted averages over the subdomain faces, edges, and vertices. We formally perform a change of basis, such that we have a splitting of the degrees of freedom (DOFs) into primal and dual ones, and work in the space $\widetilde{W}=\widehat{W}_{\Pi} \oplus W_{\Delta}$.

Let $B$ be the usual jump operator which acts on the non-primal DOFs. The FETIDP system

$$
B \widehat{K}^{-1} B^{\top} \lambda=B \widehat{K}^{-1} \widehat{f}
$$

is solved by preconditinioned conjugate gradients, where $\widehat{K}$ and $\widehat{f}$ denote the stiffness matrix and load vector partially assembled at the primal DOFs. The overall solution is then given by

$$
u=\widehat{K}^{-1}\left(\widehat{f}-B^{\top} \lambda\right) .
$$

Next, we define a FETI-DP preconditioner that is slightly modified to allow for certain coefficient jumps (cf. Klawonn and Rheinbach [2007], Pechstein and Scheichl [2009]). Let $i=1, \ldots, N$ be fixed and let $\mathscr{T}\left(\Omega_{i}\right)$ denote the mesh restricted to subdomain $\Omega_{i}$. For each mesh node $x^{h}$ on $\bar{\Omega}_{i}$, we set 


$$
\widehat{\alpha}_{i}\left(x^{h}\right):=\max _{T \in \mathscr{T}\left(\Omega_{i}\right): x^{h} \in \bar{T}} \alpha_{\mid T} .
$$

Furthermore, if $\mathscr{N}_{x^{h}}$ denotes the index set of subdomains sharing the mesh node $x^{h}$, we define the weighted counting function

$$
\delta_{i}^{\dagger}\left(x^{h}\right):= \begin{cases}\frac{\widehat{\alpha}_{i}\left(x^{h}\right)}{\sum_{j \in \mathscr{N}_{x^{h}}} \widehat{\alpha}_{j}\left(x^{h}\right)}, & \text { if } x^{h} \text { lies on } \bar{\Omega}_{i}, \\ 0, & \text { otherwise. }\end{cases}
$$

Using these counting functions we define the scaled jump operator $B_{D}$ according to [Toselli and Widlund, 2005, Sect. 6.4.1] (for details see also Pechstein and Scheichl [2011] where the same scaled jump operator was used to define a one-level FETI preconditioner). The FETI-DP preconditioner is finally given by

$$
M^{-1}:=B_{D} S_{\Delta} B_{D}^{\top},
$$

where $S_{\Delta}$ is the block-diagonal Schur complement on the non-primal interface DOFs, eliminating the interior DOFs.

\section{Weighted Poincaré inequalities with weighted averages}

Let $D$ be a bounded Lipschitz polytope and let $\left\{Y_{\ell}\right\}_{\ell=1}^{n}$ be a subdivision of $D$ into open Lipschitz polytopes such that

$$
\alpha_{\mid Y_{\ell}}=\alpha_{\ell}=\text { const. }
$$

Furthermore, let $\mathscr{X} \subset \partial D$ be a manifold of dimension $0 \leq d \mathscr{X} \leq d-1$ (usually a vertex, an open subdomain edge or an open face, or a union of these). We define

$$
\mathscr{X}_{\ell}:=\bar{Y}_{\ell} \cap \mathscr{X} .
$$

Some of these sets may be empty or have lower dimension than $\mathscr{X}$. However, with the index set $I_{\mathscr{X}}:=\left\{\ell: \operatorname{meas}_{d_{\mathscr{X}}}\left(\mathscr{X}_{\ell}\right)>0\right\}$ we can write

$$
\overline{\mathscr{X}}=\bigcup_{k \in I_{\mathscr{X}}} \overline{\mathscr{X}}_{k} .
$$

In general, for different indices $k, \ell \in I_{\mathscr{X}}$, the manifolds $\mathscr{X}_{k}$ and $\mathscr{X}_{\ell}$ may have a non-trivial intersection or even coincide. For simplicity, we assume that

$$
k \neq \ell \in I_{\mathscr{X}} \quad \Longrightarrow \quad \operatorname{meas}_{d_{\mathscr{X}}}\left(\mathscr{X}_{k} \cap \mathscr{X}_{\ell}\right)=0 .
$$

The general case needs more formalism and will be treated in an upcoming paper (Pechstein et al. [2011]). Finally, we can define a meaningful trace $\alpha_{\mathrm{tr}} \in L^{\infty}(\mathscr{X})$ of $\alpha$ by 


$$
\alpha_{\mathrm{tr}}(x)=\alpha_{k} \quad \text { for } x \in \mathscr{X}_{k}
$$

Let $\left\{V^{h}(D)\right\}_{h}$ be a family of $H^{1}$-conforming FE spaces associated with a quasiuniform family of triangulations of $D$. For $v \in V^{h}(D)$, we define the weighted (semi)norms and the weighted average on $\mathscr{X}$ by

$$
\|v\|_{L^{2}(D), \alpha}^{2}:=\int_{D} \alpha v^{2}, \quad|v|_{H^{1}(D), \alpha}^{2}:=\int_{D} \alpha|\nabla v|^{2} \quad \text { and } \quad \bar{v}^{\mathscr{X}, \alpha_{\mathrm{tr}}}:=\frac{\int_{\mathscr{X}} \alpha_{\mathrm{tr}} v}{\int_{\mathscr{X}} \alpha_{\mathrm{tr}}}
$$

We are interested in the following WPI with weighted average:

$$
\left\|u-\bar{u}^{\mathscr{X}, \alpha_{\mathrm{tr}}}\right\|_{L^{2}(D), \alpha}^{2} \leq C_{P, \alpha}(D, \mathscr{X} ; h) \operatorname{diam}(D)^{2}|u|_{H^{1}(D), \alpha}^{2} \quad \forall u \in V^{h}(D) .
$$

In particular, we are interested under which assumptions the parameter $C_{P, \alpha}(D, \mathscr{X} ; h)$ is independent of the values $\left\{\alpha_{\ell}\right\}$.

Sufficient conditions for robustness. We need two crucial assumptions for (4) to be independent of the values $\left\{\alpha_{\ell}\right\}$. The first assumption is a quasi-monotonicity assumption on $\alpha$. It has been introduced in Dryja et al. [1996] and generalized in Klawonn et al. [2002], Pechstein and Scheichl [2010]. The second assumption states that $\mathscr{X}$ "sees" the largest coefficient.

Definition 1. Let $0 \leq m<d$ and let $\ell^{*}:=\underset{1 \leq \ell \leq s}{\operatorname{argmax}} \alpha_{\ell}$ denote the index of the largest coefficient. $^{1}$

(a) We call the region $P_{\ell_{1}, \ell_{s}}:=\left(\bar{Y}_{\ell_{1}} \cup \ldots \cup \bar{Y}_{\ell_{s}}\right)^{\circ}, 1 \leq \ell_{1}, \ldots, \ell_{s} \leq n$ a type-m quasimonotone path from $Y_{\ell_{1}}$ to $Y_{\ell_{s}}$ (with respect to $\alpha$ ), if

(i) the regions $Y_{\ell_{i}}$ and $Y_{\ell_{i+1}}$ share a common $m$-dimensional manifold, and

(ii) $\alpha_{\ell_{1}} \leq \alpha_{\ell_{2}} \leq \ldots \leq \alpha_{\ell_{s}}$.

(b) We say that $\alpha$ is type-m quasi-monotone on $D$, if for all $k=1, \ldots, n$ there exists a quasi-monotone type- $m$ path from $Y_{k}$ to $Y_{\ell^{*}}$.

Assumption A2. $\alpha$ is type- $m$ quasi-monotone on $D$ for some $0 \leq m<d$.

Assumption A3. $\operatorname{meas}_{d_{\mathscr{X}}}\left(\mathscr{X} \cap \bar{Y}_{\ell^{*}}\right)>0$.

In order to formulate our main theorem, we first need some definitions of generalized Poincaré constants/parameters.

Definition 2. (i) For any bounded Lipschitz domain $Y \subset \mathbb{R}^{d}$ let $C_{P}(Y)$ be the smallest constant such that

$$
\left\|v-\bar{v}^{Y}\right\|_{L^{2}(Y)}^{2} \leq C_{P}(Y) \operatorname{diam}(Y)^{2}|v|_{H^{1}(Y)}^{2} \quad \forall v \in H^{1}(Y)
$$

\footnotetext{
${ }^{1}$ We can assume without loss of generality that $\ell^{*}$ is unique. By definition, type- $m$ quasimonotonicity implies that otherwise all maximal subregions can be combined into a single subregion.
} 
(ii) Let $Z$ be the finite union of bounded Lipschitz polytopes such that $\bar{Z}$ is connected, and let $\left\{\mathscr{T}^{h}(Z)\right\}_{h}$ be a quasi-uniform family of triangulations of $Z$ with the associated continuous piecewise linear FE spaces $\left\{V^{h}(Z)\right\}_{h}$. Let $X, W \subset \bar{Z}$ be manifolds/subdomains of (possibly different) dimension $\in\{0, \ldots, d\}$. Let $C_{P}(Z, X, W ; h)$ be the best parameter such that

$$
\left\|v-\bar{v}^{X}\right\|_{L^{2}(W)}^{2} \leq C_{P}(Z, X, W ; h) \frac{|W|}{|Z|} \operatorname{diam}(Z)^{2}|u|_{H^{1}(Z)}^{2} \quad \forall v \in V^{h}(Z) .
$$

$|W|$ and $|Z|$ denote the measures of $W$ and $Z$ (in the respective dimension).

If $Z$ is connected and if the dimensions of $X$ and $W$ are $\geq d-1$, we can define a constant $C_{P}(Z, X, W)$ independent of the discretization parameter $h$ such that the inequality in Definition 2(ii) holds for all functions in $H^{1}(Z)$.

Theorem 1. Let Assumptions $A 2$ and $A 3$ be satisfied. Then the parameter $C_{P, \alpha}(D, \mathscr{X} ; h)$ in formula (4) is independent of the values $\left\{\alpha_{\ell}\right\}_{\ell=1}^{n}$ and

$$
C_{P, \alpha}(D, \mathscr{X} ; h) \leq 2\left[C^{*, 1}(h)+C^{*, 2}(h)\right]
$$

with

$$
\begin{aligned}
C^{*, 1}(h) & :=\sum_{\ell=1}^{n} \frac{\left|Y_{\ell}\right| \operatorname{diam}\left(P_{\ell, \ell^{*}}\right)^{2}}{\left|P_{\ell, \ell^{*}}\right| \operatorname{diam}(D)^{2}} C_{P}\left(P_{\ell, \ell^{*}}, \mathscr{X}_{\ell^{*}}, Y_{\ell} ; h\right), \\
C^{*, 2}(h) & :=\frac{|D|}{\left|\mathscr{X}_{\ell^{*}}\right|} \sum_{k \in I_{\mathscr{X}}} \frac{\left|\mathscr{X}_{k}\right| \operatorname{diam}\left(P_{k, \ell^{*}}\right)^{2}}{\left|P_{k, \ell^{*}}\right| \operatorname{diam}(D)^{2}} C_{P}\left(P_{k, \ell^{*}}, \mathscr{X}_{\ell^{*}}, \mathscr{X}_{k} ; h\right) .
\end{aligned}
$$

Proof. Without loss of generality, we may assume that $\bar{u}^{\mathscr{X}, \alpha_{\mathrm{tr}}}=0$. For each index $\ell=1, \ldots, n$

$$
\frac{1}{2}\|u\|_{L^{2}\left(Y_{\ell}\right)}^{2} \leq\left\|u-\bar{u}^{\mathscr{X}_{\ell^{*}}}\right\|_{L^{2}\left(Y_{\ell}\right)}^{2}+\left|Y_{\ell}\right|\left(\bar{u}^{\mathscr{X}_{\ell^{*}}}\right)^{2} .
$$

Due to Assumption A2, there is a quasi-monotone path from $Y_{\ell}$ to $Y_{\ell^{*}}$. With $c_{\ell, \ell^{*}}:=$ $C_{P}\left(P_{\ell, \ell^{*}}, \mathscr{X}_{\ell^{*}}, Y_{\ell} ; h\right)$, summation over $\ell=1, \ldots, n$ yields

$$
\frac{1}{2}\|u\|_{L^{2}(D), \alpha}^{2} \leq \sum_{\ell=1}^{n} c_{\ell, \ell^{*}} \frac{\left|Y_{\ell}\right|}{\left|P_{\ell, \ell^{*}}\right|} \operatorname{diam}\left(P_{\ell, \ell^{*}}\right)^{2} \underbrace{\alpha_{\ell}|u|_{H^{1}\left(P_{\ell, \ell^{*}}\right)}^{2}}_{\leq|u|_{H^{1}(D), \alpha}^{2}}+\underbrace{\sum_{\ell=1}^{n} \alpha_{\ell}\left|Y_{\ell}\right|}_{\leq \alpha_{\ell^{*}}|D|}\left(\bar{u}^{\mathscr{X}_{\ell^{*}}}\right)^{2},
$$

where we have used Definition 2(ii) and the quasi-monotonicity of $P_{\ell, \ell^{*}}$. The first sum is bounded by $C^{*, 1}(h) \operatorname{diam}(D)^{2}|u|_{H^{1}(D), \alpha}^{2}$. To bound the remaining term, we use Cauchy's inequality and the definition of $\alpha_{\mathrm{tr}}$ :

$$
\alpha_{\ell^{*}}|D|\left(\bar{u}^{\mathscr{X}_{\ell^{*}}}\right)^{2} \leq \frac{|D|}{\left|\mathscr{X}_{\ell^{*}}\right|} \alpha_{\ell^{*}}\|u\|_{L^{2}\left(\mathscr{X}_{\ell^{*}}\right)}^{2} \leq \frac{|D|}{\left|\mathscr{X}_{\ell^{*}}\right|}\|u\|_{L^{2}(\mathscr{X}), \alpha_{\mathrm{tr}}}^{2} .
$$


A variational argument yields

$$
\begin{aligned}
\|u\|_{L^{2}(\mathscr{X}), \alpha_{\mathrm{tr}}}^{2} & \leq\|u-\underbrace{\bar{u}^{\mathscr{X}, \alpha_{\mathrm{tr}}}}_{=0}\|_{L^{2}(\mathscr{X}), \alpha_{\mathrm{tr}}}^{2}=\inf _{c \in \mathrm{R}}\|u-c\|_{L^{2}(\mathscr{X}), \alpha_{\mathrm{tr}}}^{2} \\
& \leq\left\|u-\bar{u}^{\mathscr{X}_{\ell^{*}}}\right\|_{L^{2}(\mathscr{X}), \alpha_{\mathrm{tr}}}^{2}=\sum_{k \in I_{\mathscr{X}}} \alpha_{k}\left\|u-\bar{u}^{\mathscr{X}_{\ell^{*}}}\right\|_{L^{2}\left(\mathscr{X}_{k}\right)}^{2} .
\end{aligned}
$$

Now, we have

$$
\alpha_{k}\left\|u-\bar{u}^{\mathscr{X}_{\ell^{*}}}\right\|_{L^{2}\left(\mathscr{X}_{k}\right)}^{2} \leq C_{P}\left(P_{k, \ell^{*}}, \mathscr{X}_{\ell^{*}}, \mathscr{X}_{k} ; h\right) \frac{\left|\mathscr{X}_{k}\right|}{\left|P_{k, \ell^{*}}\right|} \operatorname{diam}\left(P_{k, \ell^{*}}\right)^{2} \alpha_{k}|u|_{H^{1}\left(P_{k, \ell^{*}}\right)}^{2} .
$$

Using the quasi-monotonicity of $\alpha$ on $P_{k, \ell^{*}}$ finally leads to (5).

Necessity of the conditions. It was shown in [Pechstein and Scheichl, 2010, Prop. 2.11] that Assumption A2 is necessary to ensure that $C_{P, \alpha}(D, \mathscr{X} ; h)$ is independent of the values $\left\{\alpha_{\ell}\right\}$.

To see that $\mathrm{A} 3$ is necessary as well, assume that $\operatorname{meas}_{d \mathscr{X}}\left(\mathscr{X} \cap \bar{Y}_{\ell^{*}}\right)=0$. We choose a function $u$ which is one on $Y_{\ell^{*}}$. Since the average functional $v \mapsto \bar{v}^{\mathscr{X}, \alpha_{\text {tr }}}$ is independent of $\alpha_{\ell^{*}}$, we can prescribe values of $u$ on $\mathscr{X}$ such that $\bar{u}^{\mathscr{X}, \alpha_{\mathrm{tr}}}=0$ and continuously extend $u$ into $D \subset \bar{Y}_{\ell^{*}}$. The whole construction of $u$ is independent of $\alpha_{\ell^{*}}$, Since $\nabla u=0$ on $Y_{\ell^{*}}$, the seminorm $|u|_{H^{1}(D), \alpha}$ is independent of $\alpha_{\ell^{*}}$ as well. However, $\|u\|_{L^{2}(D), \alpha}^{2} \geq \alpha_{\ell^{*}}\left|Y_{\ell^{*}}\right|$. Therefore, if $\alpha \leq \alpha_{k}$ on $D \backslash Y_{\ell^{*}}$, then $C_{P, \alpha}(D, \mathscr{X} ; h)=\mathscr{O}\left(\frac{\alpha_{\ell^{*}}}{\alpha_{k}}\right)$ for $\alpha_{\ell^{*}} / \alpha_{k} \rightarrow \infty$.

This means that Assumptions A2 and A3 in some sense characterize the robustness of the WPI with weighted average.

\section{Robustness proof of FETI-DP}

To analyze the robustness of FETI-DP, we need the following assumption.

Assumption A4. For each subdomain $\Omega_{i}$ and for each subdomain edge (face) $\mathscr{E}$ of $\Omega_{i}$, there is a Lipschitz domain $D_{i, \mathscr{E}} \subset \Omega_{i}$, such that $\mathscr{E} \subset \partial D_{i, \mathscr{E}}$ and Assumptions A2 and $\mathrm{A} 3$ are satisfied for $D=D_{i, \mathscr{E}}$ and $\mathscr{X}=\mathscr{E}$. The union of all the regions $D_{i, \mathscr{E}}$ covers a boundary layer $\Omega_{i, \eta_{i}}$ of width $\eta_{i} \geq h$ of $\Omega_{i}$ (see e.g. [Pechstein and Scheichl, 2008, Def. 2.6]).

Theorem 2. Let Assumptions A1 and A4 hold. Then the condition number $\kappa\left(M^{-1} F\right)$ for the FETI-DP method is independent of the values of the coefficient $\alpha$, in particular of any non-resolved jumps.

Due to space limitations we only give a sketch of the proof. A detailed proof will be given in Pechstein et al. [2011], together with a more detailed statement of Theorem 2 that makes precise the dependence of $\kappa\left(M^{-1} F\right)$ on geometric parameters, such as the ratios $\operatorname{diam}\left(\Omega_{i}\right) / h$ and $\operatorname{diam}\left(\Omega_{i}\right) / \eta_{i}$. 
Let $\mathscr{H}_{i}$ denote the discrete $\alpha$-harmonic extension from $\partial \Omega_{i}$ to $\Omega_{i}$ and let

$$
|w|_{S}^{2}:=\sum_{i=1}^{N}\left|\mathscr{H}_{i} w\right|_{H^{1}\left(\Omega_{i}\right), \alpha}^{2}
$$

Then, following [Toselli and Widlund, 2005, Sect. 6.4.3], a bound of the kind

$$
\left|P_{D} w\right|_{S}^{2} \leq \omega|w|_{S}^{2}, \quad \text { where } \quad P_{D}:=B_{D}^{\top} B
$$

implies $\kappa\left(M^{-1} F\right) \leq \omega$.

As in the proof of [Pechstein and Scheichl, 2011, Lemma 5.6; formula (5.24)], we can introduce a set of cut-off functions associated with each subdomain edge (face) $\mathscr{E}$ whose support is contained in $D_{i, \mathscr{E}}$. It then follows that, for any $w \in \widehat{W}_{\Pi} \oplus W_{\Delta}$,

$$
\left|P_{D} w\right|_{S}^{2} \leq C \sum_{i=1}^{N}\left[\left|\mathscr{H}_{i} w_{i}\right|_{H^{1}\left(\Omega_{i}\right), \alpha}^{2}+\sum_{\mathscr{E}} \frac{1}{\operatorname{diam}\left(\Omega_{i}\right)^{2}}\left\|\mathscr{H}_{i} w_{i}-\bar{w}_{i}^{\mathscr{E}}\right\|_{L^{2}\left(D_{i, \mathscr{E}}\right), \alpha}^{2}\right]
$$

where $C$ depends on $\operatorname{diam}\left(\Omega_{i}\right) / h$ and $\operatorname{diam}\left(\Omega_{i}\right) / \eta_{i}$, but it is independent of the values $\left\{\alpha_{\ell}\right\}$. By Theorem 1 , we can bound each of the weighted $L^{2}$ norms by the weighted $H^{1}$ seminorm of $\mathscr{H}_{i} w_{i}$, and thus obtain (6).

\section{Numerical results}

We provide results for the three examples shown in Figure 2. Note that in the last example, the coefficient is not quasi-monotone on one of the subdomains, but satisfies Assumptions A1 and A4. The estimated condition numbers and the number of PCG iterations are displayed in Table 1 . They clearly confirm Theorem 2.
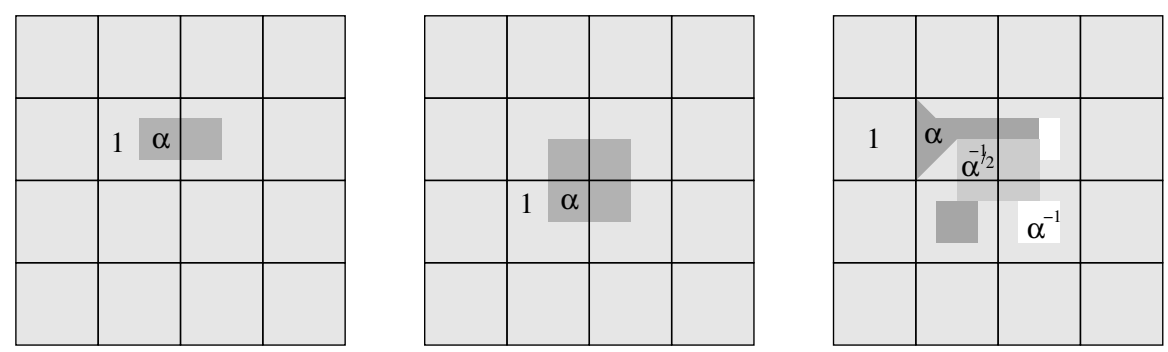

Fig. 2 Edge-island (left), cross-point island (middle), complicated coefficient (right).

Acknowledgment. The authors would like to thank Clark Dohrmann for the fruitful discussions during and after the DD20 conference. 


\begin{tabular}{ccc}
$\alpha$ & condition $\#$ iterations \\
\hline 1 & 1.58 & 10 \\
\hline $10^{1}$ & 1.57 & 10 \\
$10^{3}$ & 1.56 & 10 \\
$10^{5}$ & 1.56 & 10 \\
$10^{7}$ & 1.56 & 10 \\
\hline $10^{-1}$ & 1.70 & 10 \\
$10^{-3}$ & 1.74 & 10 \\
$10^{-5}$ & 1.74 & 10 \\
$10^{-7}$ & 1.74 & 11
\end{tabular}

\begin{tabular}{ccc}
$\alpha$ & condition & \#iterations \\
\hline 1 & 1.58 & 10 \\
\hline $10^{1}$ & 1.59 & 10 \\
$10^{3}$ & 1.59 & 10 \\
$10^{5}$ & 1.59 & 10 \\
$10^{7}$ & 1.59 & 10 \\
\hline $10^{-1}$ & 1.57 & 10 \\
$10^{-3}$ & 1.57 & 10 \\
$10^{-5}$ & 1.57 & 10 \\
$10^{-7}$ & 1.57 & 10
\end{tabular}

\begin{tabular}{ccc}
$\alpha$ & condition & \#iterations \\
\hline 1 & 1.58 & 10 \\
\hline $10^{1}$ & 1.61 & 11 \\
$10^{2}$ & 1.62 & 11 \\
$10^{3}$ & 1.62 & 11 \\
$10^{4}$ & 1.62 & 11 \\
\hline $10^{-1}$ & 1.62 & 11 \\
$10^{-2}$ & 1.60 & 11 \\
$10^{-3}$ & 1.59 & 11 \\
$10^{-4}$ & 1.59 & 11
\end{tabular}

Table 1 Edge-island (left), crosspoint-island (middle), complicated coefficient (right), $H / h=32$.

\section{References}

M. Dryja, M. V. Sarkis, and O. B. Widlund. Multilevel Schwarz methods for elliptic problems with discontinuous coefficients in three dimensions. Numer. Math., 72 (3):313-348, 1996.

C. Farhat, M. Lesoinne, P. Le Tallec, K. Pierson, and D. Rixen. FETI-DP: a dualprimal unified FETI method. I. A faster alternative to the two-level FETI method. Internat. J. Numer. Methods Engrg., 50(7):1523-1544, 2001.

A. Klawonn and O. Rheinbach. Robust FETI-DP methods for heterogeneous three dimensional elasticity problems. Comput. Methods Appl. Mech. Engrg., 196(8): 1400-1414, 2007.

A. Klawonn, O. B. Widlund, and M. Dryja. Dual-primal FETI methods for threedimensional elliptic problems with heterogeneous coefficients. SIAM J. Numer. Anal., 40(1):159-179, 2002.

J. Mandel and R. Tezaur. On the convergence of a dual-primal substructuring method. Numer. Math., 88(3):543-558, 2001.

C. Pechstein and R. Scheichl. Analysis of FETI methods for multiscale PDEs. $\mathrm{Nu}$ mer. Math., 111(2):293-333, 2008.

C. Pechstein and R. Scheichl. Scaling up through domain decomposition. Appl. Anal., 88(10):1589-1608, 2009.

C. Pechstein and R. Scheichl. Weighted Poincaré inequalities. NuMa Report 2010-10, Institute of Computational Mathematics, JKU Linz, 2010. Submitted, available at www.numa.uni-linz.ac.at/Publications/List/ 2010/2010-10.pdf.

C. Pechstein and R. Scheichl. Analysis of FETI methods for multiscale PDEs - part II: interface variation. Numer. Math., 2011. Online first February 21, 2011, DOI: 10.1007/s00211-011-0359-2.

C. Pechstein, M. Sarkis, and R. Scheichl. Analysis of FETI-DP methods for multiscale PDEs. In preparation, 2011.

A. Toselli and O. Widlund. Domain Decomposition Methods - Algorithms and Theory, volume 34 of Springer Series in Computational Mathematics. SpringerVerlag, Berlin, 2005. 